FM 20:

Astronomy for Development 


\title{
Preface - Focus Meeting 20: Astronomy for Development
}

\author{
Kevin Govender \\ IAU Office of Astronomy for Development, Cape Town, South Africa \\ email: kg@astro4dev.org
}

\section{Overview}

This Focus Meeting was about the global developmental impact that all aspects related to astronomy can deliver. The interdisciplinary nature of the meeting made it relevant to all IAU Divisions and the professional astronomy community in general. The manner in which the strategic plan has been designed and the way in which OAD implements it allows for input and innovation from the professional community both to develop the astronomy field globally and to stimulate the developmental benefits arising from the astronomy field. IAU members have played a key role in every stage of implementation of the strategic plan, from its ratification, through to strong participation in its implementation. This meeting served to report back to them in terms of progress, as well as seek input from them in terms of shaping the way forward.

\section{Structure}

The programme was organised to try to cover the wide variety of topics related to the OAD within a very limited amount of time allocated. The first session was for overview talks covering the history, achievements and activities of the OAD. This was followed by three sessions for the Task Forces (Universities and Research, Children and Schools, Public Outreach). We then had contributions from the Regional Offices and Language Expertise Centres, followed by a Poster Plug Session (where poster contributors could say a few words to attract people to their posters). The final session comprised a Panel discussion with Representatives from IAU Divisions, and an unconference session which sought topics from participants during the General Assembly in order to adapt to popular demand. These Proceedings reflect this general structure with a table summary at the end listing poster contributors' topics and authors. Presentations from the Focus Meeting, and any related information can be found on the OAD website www.astro4dev.org.

\section{Acknowledgements}

We are grateful to the IAU for the opportunity to conduct this Focus Meeting at the 2015 General Assembly and to the SOC for their voluntary efforts in driving the event before, during and after. A special word of thanks to the OAD staff, Visiting Fellows and Interns who handle all the logistics and detail required behind the scenes to ensure smooth implementation of such activities, including assistance with compiling these proceedings. Lastly, and most importantly, we wish to acknowledge and appreciate the contributions from our many collaborators (both old and new) from around the world. Without your Oral and Poster contributions, this Focus Meeting would not have happened at all, let alone with the incredible amount of enthusiasm, energy and positive feedback we experienced before, during and after the event. 